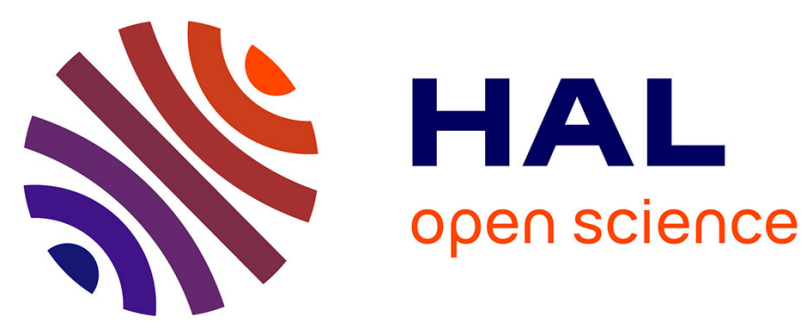

\title{
A new method for the in vivo identification of mechanical properties in arteries from cine MRI images: theoretical framework and validation.
}

Alexandre Franquet, Stéphane Avril, Rodolphe Le Riche, Pierre Badel, Fabien Schneider, Zhi-Yong Li, Chrisitan Boissier, Jean-Pierre Favre

\section{To cite this version:}

Alexandre Franquet, Stéphane Avril, Rodolphe Le Riche, Pierre Badel, Fabien Schneider, et al.. A new method for the in vivo identification of mechanical properties in arteries from cine MRI images: theoretical framework and validation.. IEEE Transactions on Medical Imaging, 2013, 32 (8), pp.1448 - 1461. 10.1109/TMI.2013.2257828. hal-00805124

\section{HAL Id: hal-00805124 https://hal.science/hal-00805124}

Submitted on 27 Mar 2013

HAL is a multi-disciplinary open access archive for the deposit and dissemination of scientific research documents, whether they are published or not. The documents may come from teaching and research institutions in France or abroad, or from public or private research centers.
L'archive ouverte pluridisciplinaire HAL, est destinée au dépôt et à la diffusion de documents scientifiques de niveau recherche, publiés ou non, émanant des établissements d'enseignement et de recherche français ou étrangers, des laboratoires publics ou privés. 


\title{
A new method for the in vivo identification 2 of mechanical properties in arteries from cine MRI images: theoretical framework and validation.
}

Alexandre Franquet, Stéphane Avril, Rodolphe Le Riche, Pierre Badel, Fabien C Schneider, Zhi Yong Li, Christian Boissier and Jean Pierre Favre

1. A. Franquet, S. Avril and P. Badel are with the CIS-EMSE, CNRS UMR 5146, Ecole Nationale Supérieure des Mines, Saint-Etienne F-42023, France. e-mail: avril@emse.fr

2. R. Le Riche is with CNRS UMR 6158 and the Ecole nationale Supérieure des Mines, Saint-Etienne F-42023

3. Z.Y. Li is with the School of Biological Science and Medical Engineering, Southeast University, Nanjing 210096, China

4. F.C. Schneider, C. Boissier and J.P. Favre are with the Radiology Department, Vascular Department, and Cardiovascular surgery Department, respectively, University Hospital of Saint Etienne France and GRTPAR UR4672 Jean Monnet University, Saint Etienne F-42000, France.

This work has been submitted to the IEEE for possible publication. Copyright may be transferred without notice, after which this version may no longer be accessible

\begin{abstract}
Quantifying the stiffness properties of soft tissues is essential for the diagnosis of many cardiovascular diseases such as atherosclerosis. In these pathologies it is widely agreed that the arterial wall stiffness is an indicator of vulnerability. The present paper focuses on the carotid artery and proposes a new inversion methodology for deriving the stiffness properties of the wall from cine-MRI (Magnetic Resonance Imaging) data. We address this problem by setting-up a cost function defined as the distance between the modeled pixel signals and the measured ones. Minimizing this cost
\end{abstract}




\begin{abstract}
function yields the unknown stiffness properties of both the arterial wall and the surrounding tissues. The sensitivity of the identified properties to various sources of uncertainty is studied. Validation of the method is performed on a rubber phantom. The elastic modulus identified using the developed methodology lies within a mean error of $9.6 \%$. It is then applied to two young healthy subjects as a proof of practical feasibility, with identified values of $625 \mathrm{kPa}$ and $587 \mathrm{kPa}$ for one of the carotid of each subject.
\end{abstract}

Keywords Finite elements, mechanical properties, elasticity, inverse method, identification, artery, in vivo analysis.

\title{
1 Introduction
}

Quantifying the stiffness properties of soft tissues is essential for the diagnosis of many diseases. This is especially important for cardiovascular pathologies where it has been shown that the stiffness of the arterial wall is an indicator of the stroke risk for diseases such as atherosclerosis [1]. This could be used to improve the diagnosis in clinical practice.

The mechanical properties of arteries have been a research topic of major attention for thirty years $([2,3,4,5,6,7,8,9,10])$. Many authors have characterized the mechanical properties of excised arteries using either tensile tests, indentation tests or inflation tests. However the results reported in the literature are not very consistent. The large scattering observed can be attributed to the differences in experimental conditions and conservation processes, to the anisotropic and behavioral non linearities $([5,11,12])$, or to the natural interindividual variability.

Therefore, the identification of the mechanical properties in vivo provides another essential point of view. In vivo identification techniques are more constraining because they are usually non-intrusive. An overview of the existing methods and results of identification is presented in Table 1 which will be thoroughly discussed in the "Discussion". In clinical applications, for assessing arterial stiffness, it is still commonplace to use the Moens-Korteweg theoretical equation relating the Pulse Wave Velocity and the incremental modulus in a round cylinder [13]. Recent developments based on the propagation of shear waves in soft tissues have enabled clinicians to improve the evaluation of the arterial stiffness [14]. Although these types of methods are well suited for rapid 
medical examinations, they suffer, among other things, from a poor precision because of the strong assumptions made about the mechanical behavior of arteries.

In order to improve precision, medical imaging techniques can be employed. The basic method consists in tracking the diameter changes in the images and relating these to blood pressure variations $([15,16,17,18,19,20,21,22,23,24$, $25,14])$. The advantage is that a non-linear behavior can possibly be identified [26] however the precision remains questionable due to the assumptions about the geometry of the artery.

Imaging techniques providing access to kinematic fields (displacements, strains) offer an attractive flexibility and enable the identification of more complex mechanical behaviors with a limited number of mechanical assumptions. However, they require the development of appropriate inverse methods [27]. The most natural one is the Finite Element Model Updating (FEMU) method which has been applied several times to data acquired in vivo.

The FEMU method consists in finding the parameters of a Finite Element (FE) model that minimize the gap between numerical and experimental kinematic fields (displacements, strains). This method has been successfully applied to identify the heterogeneous elastic properties of a pig artery with an atherosclerotic plaque $([28,29])$. The elastic properties and the contours of an atherosclerotic plaque in the human coronary artery were also determined in a similar way using a Parametric FEMU method (P-FEMU) [30]. Using the same idea, other authors [31] have designed a specific algorithm where the contours are recovered by a watershed method and the heterogeneous elastic properties are found at the same time.

All these applications of the FEMU method are based on experimental strain fields obtained by IntraVascular UltraSounds (IVUS). IVUS is an ultrasonic imaging technique where the ultrasound probe is inserted inside the arterial conduit using a catheter. Specific algorithms have been developed for deriving radial strains from the Radio Frequency (RF) signals with a suitable spatial resolution (pixel size $\sim 0.1 \mathrm{~mm}$ ). But the IVUS technique is very invasive due to the use of catheters thereby limiting its applications.

Other non-invasive imaging techniques are available for mapping the strain fields but their spatial resolution is inferior. For instance, strain maps can be measured in vivo in the vicinity of the carotid artery using a specific Mag- 
netic Resonance Imaging (MRI) sequence: the DENSE (Displacement ENcoding with Stimulated Echoes) sequence [32]. The resulting pixel size is over $0.5 \mathrm{~mm}$ and moreover requires highly skilled operators.

Despite the great potential of MRI for these types of problems, the pixel size is usually not fine enough to track the strain fields of the arterial wall, limiting the use of MRI for the purpose of accurately identifying the mechanical behavior of arterial walls.

In this paper we address this issue by setting-up a model relating the temporal variations of the pixel MR signal to the strains and deformations of the tissue. Using this approach, we are able to combine the advantages of a full field based identification method with a non invasive imaging device. A novel inverse method is developed called Magnitude-Based FEMU (MB-FEMU). The cost function is defined as the distance between the modeled pixel signals and the measured ones. Minimizing this cost function yields the unknown stiffness properties of the arterial wall and possibly of the surrounding tissues. This type of method has been developed in the context of elastography $([33,34])$ for assessing hard nodules in breast cancers. However the issue of the low spatial resolution of MRI compared with the size of the specimen may prevent from using this method on arteries. In this study we used the partial volume effect that occurs in MRI, which corresponds to the partial filling of the pixels by different materials.

After presenting the theoretical and numerical foundations of the method, the method is validated on a rubber phantom and then applied to two subjects as a proof of practical feasibility.

\section{Methodology}

The approach is based on the minimization of a cost function which measures the distance between a template image and a registered image. The template image is the experimental image of a deformed artery provided by the magnitude of a cine Phase-Contrast MR sequence. The registered image is obtained from the experimental image of the undeformed artery and a displacement field provided by a FE analysis. After introducing the theoretical framework, the implementation of the method is described and then the experimental set up used to validate the method is presented. 
Table 1: Literature summary of methods, imaging systems and Young's moduli of arteries identified in vivo.

\begin{tabular}{|c|c|c|c|c|c|c|}
\hline Team & Year & Artery & Imaging system & $\begin{array}{l}\text { Number of } \\
\text { subjects }\end{array}$ & Methodology/index & Value $(\mathrm{kPa})$ \\
\hline Riley et al. & 1992 & $\begin{array}{l}\text { Common } \\
\text { carotid }\end{array}$ & B-mode echo & 419 & Variation of diameter & $701 \pm 324$ \\
\hline Laurent et al. & 1994 & $\begin{array}{l}\text { Radial } \\
\text { artery }\end{array}$ & RF US signal & 22 & $\begin{array}{l}\text { Variation of diameter } \\
\qquad / E_{\text {inc }}\end{array}$ & $2680 \pm 1810$ \\
\hline Brands et al. & 1999 & $\begin{array}{l}\text { Common } \\
\text { carotid }\end{array}$ & RF US signal & 1 & $\begin{array}{l}\text { Variation of diameter } \\
\qquad / E_{\text {inc }}\end{array}$ & 480 \\
\hline Aggoun et al. & 2000 & $\begin{array}{l}\text { Common } \\
\text { carotid }\end{array}$ & B-mode echo & 21 & $\begin{array}{l}\text { Variation of diameter } \\
\qquad / E_{i n c}\end{array}$ & $187 \pm 67$ \\
\hline Bussy et al. & 2000 & $\begin{array}{l}\text { Common } \\
\text { carotid }\end{array}$ & RF US signal & 40 & $\begin{array}{c}\text { Variation of diameter } \\
\qquad / E_{i n c}\end{array}$ & $710 \pm 290$ \\
\hline Boutouyrie et al. & 2001 & $\begin{array}{l}\text { Common } \\
\text { carotid }\end{array}$ & RF US signal & 15 & $\begin{array}{c}\text { Variation of diameter } \\
\qquad / E_{\text {inc }}\end{array}$ & $431 \pm 243$ \\
\hline Selzer et al. & 2001 & $\begin{array}{l}\text { Common } \\
\text { carotid }\end{array}$ & B-mode echo & 24 & Variation of diameter & $630 \pm 230$ \\
\hline Kanai et al. & 2003 & $\begin{array}{l}\text { Common } \\
\text { carotid }\end{array}$ & RF US signal & 2 & Variation of diameter. & 960 \\
\hline Boutouyrie et al. & 2004 & $\begin{array}{l}\text { Common } \\
\text { carotid }\end{array}$ & RF US signal & 16 & $\begin{array}{l}\text { Variation of diameter } \\
\qquad / E_{\text {inc }}\end{array}$ & $230 \pm 110$ \\
\hline $\begin{array}{l}\text { Hasegawa and } \\
\text { Kanai }\end{array}$ & 2004 & $\begin{array}{l}\text { Common } \\
\text { carotid }\end{array}$ & RF US signal & 1 & Variation of diameter & 1400 \\
\hline Baldewsing et al. & 2005 & $\begin{array}{l}\text { Coronary } \\
\text { artery }\end{array}$ & IVUS & 1 & Strain based FEMU & 188 \\
\hline Taviani et al. & 2008 & $\begin{array}{l}\text { Common } \\
\text { carotid }\end{array}$ & MRI & 3 & $\begin{array}{c}\text { Lumen's area based } \\
\text { FEMU }\end{array}$ & 250 \\
\hline Avril et al. & 2009 & $\begin{array}{l}\text { Common } \\
\text { carotid }\end{array}$ & MRI & 1 & Pulse wave Analysis & 99 \\
\hline Couade et al. & 2010 & $\begin{array}{l}\text { Common } \\
\text { carotid }\end{array}$ & RF US signal & 1 & $\begin{array}{l}\text { Shear waves } \\
\text { propagation }\end{array}$ & 402 \\
\hline
\end{tabular}

\subsection{Theoretical framework}

\subsubsection{Kinematics principles}

In continuum mechanics a body can be viewed as a set of elements called material points that occupy regions in space. The reference and current configurations of a body refer respectively to the undeformed or initial configuration of the body, denoted $\mathcal{C}_{0}$, and to the deformed or current configuration, denoted $\mathcal{C}_{t}$, after the application of forces and moments. The position of a material point in the initial configuration $\mathcal{C}_{0}$ is written $\underline{X}$ while the position of a material point in the current configuration $\mathcal{C}_{t}$ is written $\underline{x} . \underline{x}$ is a function of the coordinates of 
the material points and of time such as $\underline{x}=\Phi_{\theta}(\underline{X})=\underline{X}+\underline{U}(\underline{X})$. Similarly it is possible to write $\underline{X}=\Phi_{\theta}^{-1}(\underline{x})=\underline{x}-\underline{U}(\underline{X})$ where the transformation $\Phi_{\theta}$ is a function of the material properties $\theta$ and of $U$ the displacement. It is possible to introduce the function $u$ which will be used in this paper so that $\underline{X}$ is a function of $\underline{x}$ only:

$$
\begin{array}{rlc}
\underline{X} & = & \underline{x}-\underline{U}(\underline{X}) \\
& = & \underline{x}-\underline{U}\left(\Phi_{\theta}^{-1}(\underline{x})\right) \\
& = & \underline{x}-\underline{u}(\underline{x})
\end{array}
$$

\subsubsection{Signal magnitude in MRI}

In medical imaging, contrast results from materials which are different in nature. A cine-MRI sequence allows to measure the structure's response at different times of the cardiac cycle. We assumed that the magnitude at position $\underline{x}$ of the signal in the deformed configuration $\mathcal{C}_{t}$ is identical to the magnitude of the signal at position $\Phi_{\theta}^{-1}(\underline{x})$ in the initial configuration $\mathcal{C}_{0}$. This is justified by the fact that $\underline{x}$ and $\Phi_{\theta}^{-1}(\underline{x})$ are the positions of the same material particle in two different configurations. This means that:

$$
I(\underline{x})=I_{0}\left(\Phi_{\theta}^{-1}(\underline{x})\right)
$$

where $I$ is the magnitude in the current configuration $\mathcal{C}_{t}$ and $I_{0}$ is the magnitude in the initial configuration $\mathcal{C}_{0}$. An implementation of $\Phi_{\theta}$ will be proposed later in the present article based on FE displacement fields.

\subsubsection{Identification of material properties}

Equation (2) is verified if the transformation $\Phi_{\theta}$ corresponds to the real experimental transformation. Part of the inverse method is based on the evaluation of a cost function which calculates the distance between a measured quantity and an estimation of this quantity which is a function of unknown parameters. In our case the transformation $\Phi_{\theta}$ of the structure is unknown. It is affected by the material parameters which can therefore be identified by minimizing the following distance or cost function $J_{2}$ :

$$
J_{2}(\theta)=\frac{1}{2} \iint_{\Omega}\left(I(\underline{x})-I_{0}\left(\Phi_{\theta}^{-1}(\underline{x})\right)\right)^{2} d \underline{x}
$$


The domain $\Omega$ corresponds to the imaged body. $I_{0}$ and $I$ are experimental images measured by the imaging system in the configurations $\mathcal{C}_{0}$ and $\mathcal{C}_{t}$, respectively. An important feature of our method is that the distance $J_{2}$ is directly expressed in terms of image magnitudes, as opposed to displacements, strains, stresses, or energies.

Minimizing Equation (3) on $\theta$ gives the material properties for which the image associated to the transformation $\Phi_{\theta}$ is the closest to the measured image.

\subsection{Implementation}

The proposed MB-FEMU methodology is an instance of the Finite Element Model Updating method. This method can be described by the association of: (i) experimental data, (ii) a numerical model based on finite elements and (iii) a cost function which measures the distance between (i) and (ii). MRI was chosen for providing the experimental data. The numerical model consists in computing the image of the deformed geometry from displacement fields which were estimated by a FE computation. The cost function is an elaboration of Equation (3). These three aspects are now further detailed. Later in the article, the method is applied in vitro to a rubber phantom and in vivo to two healthy subjects.

\subsubsection{Experimental data}

A Phase Contrast sequence was used to provide cine-MRI data [35]. A bipolar magnetic gradient is applied to the imaged body during the excitation phase which means that a first gradient is applied along one direction followed by the application of a second gradient in the opposite direction during the same time frame. The moving protons and the stationary protons are respectively dephased and rephased by the second gradient. The phase information of this sequence is often used to obtain the flow velocity in the out-of-plane direction. In our case however, only the magnitude of the signal is considered since it is mainly correlated to the $T_{1}$ intrinsic property of the materials.

\subsubsection{Numerical model}

Finite element modeling was chosen to model the structure (phantom and arteries). 2D plane strain was assumed inasmuch as out-of-plane displacements 
were small compared to in-plane displacements and MR slice thickness. Quasi static conditions were used as heart beats have a low frequency (approximately $1 \mathrm{~Hz}$ ). In the loading interval, only the tangent behavior of both the phantom and the arteries was considered: linear elasticity in the finite deformations framework was assumed for all materials.

\subsubsection{Cost function description}

The FEMU process iteratively adjusts the mechanical parameters of a model based on its mechanical response as obtained by solving FE models until convergence is reached. The iterations are created by the minimization of a distance with respect to the parameters:

$$
\min _{\theta} J_{2}(\theta)
$$

In practice the spatial resolution of MRI depends on the acquisition time, which should be small enough to satisfy the constraints of a clinical implementation, and on the number of $\underline{k}$ sets which can be measured. Equation (3) is then a discrete sum with $N$ terms (= number of pixels). In order to save computation time we assumed that the pixels were undeformable and that the transformation of a pixel is a translation only. As a consequence, the inverse transformation $\Phi_{\theta}^{-1}$ can be simplified for a pixel centered at coordinates $\xi_{k}$ as:

$$
\Phi_{\theta}^{-1}\left(\underline{\xi_{k}}\right) \approx \underline{\xi_{k}}-\underline{u_{\theta}}\left(\underline{\xi_{k}}\right)
$$

where $u_{\theta}$ is the displacement in the current configuration $\mathcal{C}_{t}$ (see notation introduced in Equation (1)) which depends on the mechanical properties $\theta$.

Fig. 1 describes the calculation of the signal magnitude provided by the pixel $k$ which, following the above assumptions, is a discrete sum:

$$
I_{0}\left(\Phi_{\theta}^{-1}\left(\underline{\xi_{k}}\right)\right) \approx I_{0}\left(\underline{\xi_{k}}-\underline{u_{\theta}}\left(\underline{\xi_{k}}\right)\right)=\sum_{j=1}^{4} \alpha_{k j}(\theta) \cdot I_{0}\left(\underline{\xi_{k j}}\right)
$$

As illustrated in Fig. $1, k 1, \ldots, k 4$ are the pixels which, in the current configuration, intersect the initial image $I_{0}\left(\xi_{k j}\right) . \alpha_{k 1}, \ldots, \alpha_{k 4}$ are the normalized intersection areas.

The whole image can be reconstructed in this way. However, in order to provide more sensitivity to the method, a restrained set of pixels called "control 


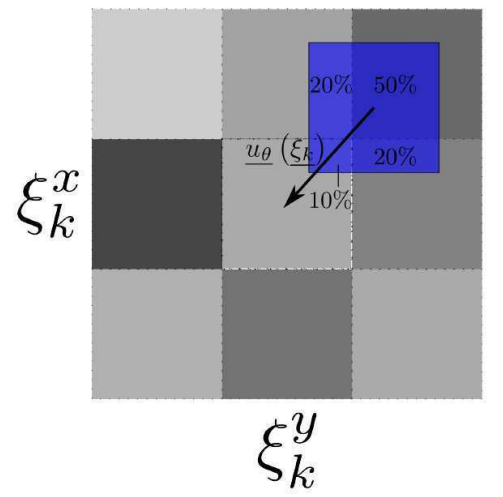

(a) Initial image

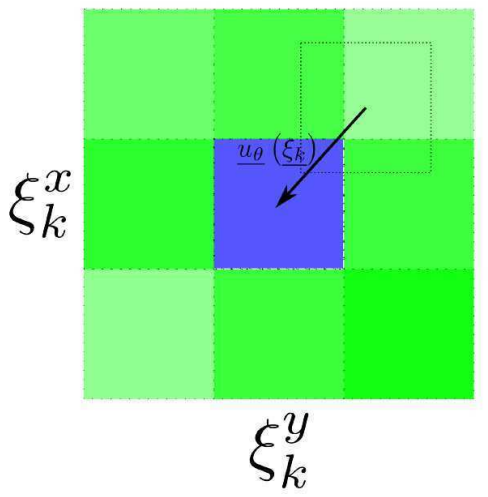

(b) Registered image

Figure 1: Illustration of the principle applied to generate the current image from the reference image and the computed displacements $u_{\theta}\left(\underline{\xi}_{k}\right)$ in order to recover the coefficients $\alpha_{k j}$ for each pixel. In this example, the magnitude of the pixel in the middle of the deformed image is calculated using the coefficients $\left\{\alpha_{k 1}, \alpha_{k 2}, \alpha_{k 3}, \alpha_{k 4}\right\}=\{50 \%, 20 \%, 10 \%, 20 \%\}$ (see Equation 6).

pixels" was selected. Control pixels were located in areas where a large change in signal magnitude is likely to be observed i.e. at the lumen contours. A binary mask is defined to select the control pixels (see Fig. 2a) so that $N$ is less than the total number of pixels. The cost function is normalized by the number of pixels considered.

Finally, the cost function is written as:

$$
\begin{aligned}
J_{2}(\theta) & =\frac{1}{2 \cdot N} \sum_{k=1}^{N}\left(I\left(\underline{\xi_{k}}\right)-I_{0}\left(\Phi_{\theta}^{-1}\left(\underline{\xi_{k}}\right)\right)\right)^{2} \\
& =\frac{1}{2 \cdot N} \sum_{k=1}^{N}\left(I\left(\underline{\xi_{k}}\right)-\sum_{j=1}^{4} \alpha_{k j}(\theta) \cdot I_{0}\left(\underline{\xi_{k j}}\right)\right)^{2}
\end{aligned}
$$

The minimization of Equation (7) is an iterative process where different combinations of material parameters $\theta$ were tested in a FE model. The updated vector of parameters $(\theta \leftarrow \theta+\Delta \theta)$ was determined by a Levenberg-Marquardt algorithm with bounds handling [36]. It requires gradients of the computed intensity $I_{0}\left(\Phi_{\theta}^{-1}\left(\xi_{k}\right)\right)$ with respect to the parameters $\theta$ which were estimated by backward finite differences (or forward differences at lower bounds). The identification process stopped when one of the following termination criteria was satisfied: $J_{2}(\theta) \leq \mathcal{E}_{a}=10^{-7}$ (accuracy on $J_{2}$ was reached) or $\|\Delta \theta\| \leq \varepsilon_{b}=$ $10^{-3}$ (the step size was too small, no more improvement was expected.). We 


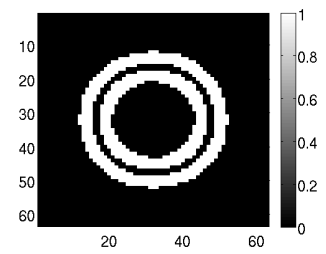

(a) Binary mask which highlights the control pixels.

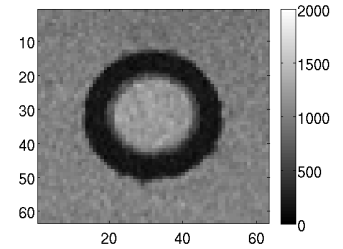

(b) Initial filtered image $I_{0}\left(\underline{\xi_{k}}\right)$.

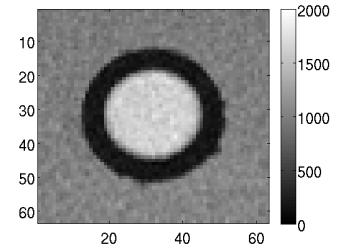

(c) Deformed filtered image $I\left(\underline{\xi_{k}}\right)$.

Figure 2: Example of images processing during the phantom study. A mask is defined to select the control pixels.

previously showed the importance of setting these two criteria ([37]).

The method is summarized in the flow chart of Fig. 3.

\subsection{Validation of the MB-FEMU method}

\subsubsection{Experimental subjects}

Phantom description An arterial rubber phantom was used to perform in vitro experimentation. The phantom was a $50-\mathrm{cm}$-long tube with a $7 \mathrm{~mm}$ inner diameter and $10 \mathrm{~mm}$ outer diameter made of PVA gel (1 freeze-thaw-cycle), surrounded by gadolinium enriched water mimicking the surrounding tissue (see Fig. 4). A peristaltic pump generated a pulsating flow mimicking physiological pressure while the wall motion was imaged.

Patients description Two healthy human subjects were selected for the experiment. They were 25-year-old and 24-year-old healthy subjects $(185 \mathrm{~cm} / 85 \mathrm{~kg}$ and $183 \mathrm{~cm} / 80 \mathrm{~kg}$, respectively). Their common carotid arteries were imaged using MRI. 


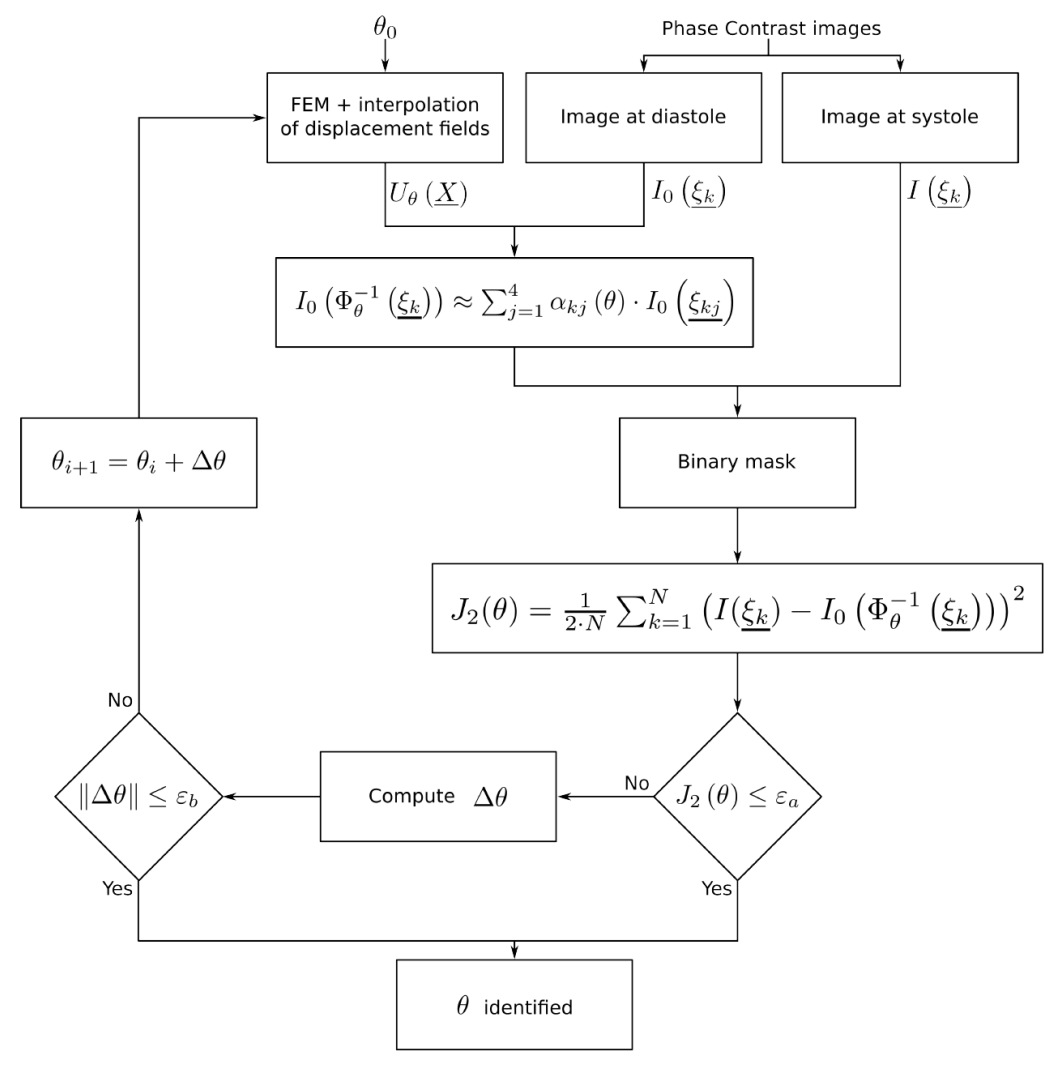

Figure 3: Flow chart of the MB-FEMU methodology to identify mechanical and other parameters. From the cine Phase Contrast MRI images, initial (diastole) and deformed (systole) images were chosen. A FE model is created from the initial image. Then for a given set of initial parameters $\theta_{0}$ a FE model is computed to provide a displacement field $U_{\theta}(\underline{X})$. This field is applied to the initial image $I_{0}\left(\underline{\xi_{k}}\right)$ according to the method described in the text in order to generate a registered image, $I_{0}\left(\Phi_{\theta}^{-1}\left(\underline{\xi}_{k}\right)\right)$. The cost function $J_{2}$ is the distance between this registered image and the experimental deformed image, calculated for a given region of interest (binary mask: control pixels). A Levenberg-Marquardt with bounds handling is used to minimize the cost function $J_{2}$ with respect to the parameters $\theta$. The procedure stops if the desired accuracy on $J_{2}$ is reached $\left(\varepsilon_{a}\right)$ or if the step size is too small $\left(\varepsilon_{b}\right)$ to expect significant further improvements of the cost function $J_{2}$.

\subsubsection{Reference data}

Measurement technique A 3T Siemens Verio system MRI scanner was used 


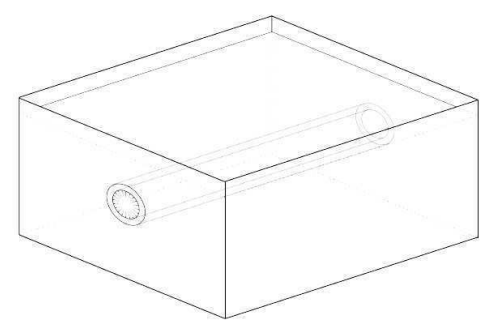

(a) The $500 \mathrm{~mm}$ long PVA phantom was surrounded by water. A maximum pressure of $70 \mathrm{mmHg}$ was applied to the tube's lumen.

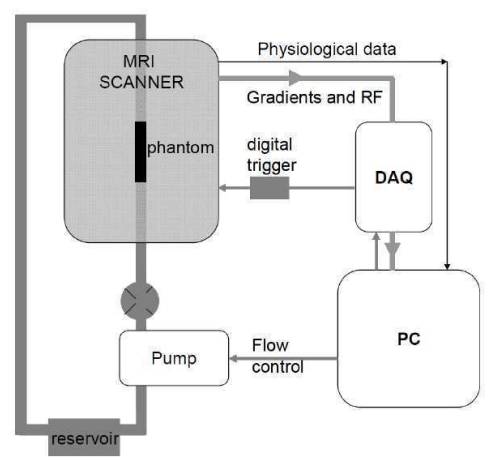

(b) Experimental set-up (Diagram not to scale).

Figure 4: Description of the rubber phantom experiment.

single $3 \mathrm{~mm}$ thick slice with a matrix size of $256 \times 256$ giving in-plane dimensions $0.27 \mathrm{~mm} \times 0.27 \mathrm{~mm}$ for the in vitro experiments and $0.586 \mathrm{~mm} \times 0.586 \mathrm{~mm}$ for the in vivo study. The image acquisition was synchronized either with the signal command for the phantom or with the physiological signal for the subjects. Regarding the physiological signal, infrared spectroscopy was used to measure the blood flow in one of the subject's finger. We obtained $N_{t}=50$ and $N_{t}=43$ snapshots for the phantom and for the subjects respectively, evenly distributed throughout the cardiac cycle. It corresponds to a mean sampling frequency of $50 \mathrm{~Hz}$ and $40 \mathrm{~Hz}$, respectively. The magnitude of the signal was digitized with a 12 bits resolution (integer between 0 and 4 095).

Choice of experimental images The raw magnitude image was a $3 \mathrm{D}$ array of size $256 \times 256 \times N_{t}$, denoted $I_{n}^{\text {raw }}\left(\underline{\xi_{l}}\right)$ for $l$ varying from 0 to 65535 , and $n$ varying from 0 to $N_{t}-1$. The images were filtered along the time axis using a Gaussian filter with a kernel size $\sigma=1.5$ to produce $I_{n}\left(\underline{\xi_{k}}\right)$.

A region of interest of $63 \times 63$ pixels was selected around the vessel. The first image $I_{0}\left(\underline{\xi_{k}}\right)$ with $k$ varying from 0 to 3968 was chosen as the initial image. The deformed image was selected through the images $I_{n}\left(\underline{\xi_{k}}\right)$ for $1 \leq$ $n \leq N_{t}-1$ according to the following definition:

$$
\max \left(\overline{\left|I_{n}\left(\underline{\xi_{k}}\right)-I_{0}\left(\underline{\tilde{\xi}_{k}}\right)\right|}\right)
$$


An example of experimental images is shown in Fig. 5.

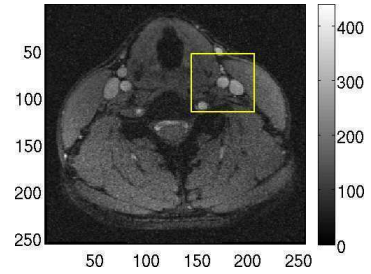

(a) Raw global image. The yellow square is a region of interest of size $63 \times 63$ pixels.

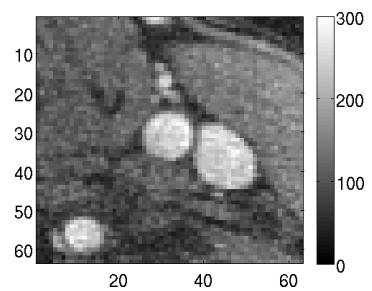

(b) Region of interest around the common carotid artery. The jugular vein touches the carotid artery. The vertebral artery can be observed at the bottom left.

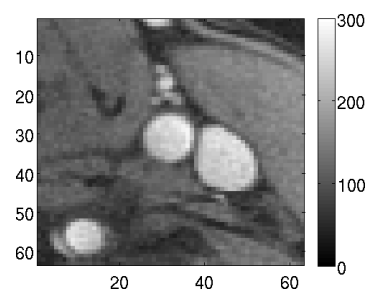

(c) Image filtered with a

Gaussian temporal smoothing filter. The kernel size (standard deviation) is 1.5 .

Figure 5: Example of a magnitude image obtained by PC MRI. The raw image is filtered after having chosen a region of interest.

Choice of control pixels A binary mask was defined before the identification procedure to discriminate the control pixels (see Fig. 2): (i) pixels which contain the moving contours were selected automatically. (ii) Additional control pixels were added by dilating the previous binary mask according to:

$$
M_{(i i)}=M_{(i)} \oplus S
$$

where $M_{(i i)}$ is the final binary mask used; $M_{(i)}$ is the binary mask at step (i); and $S$ is a square structuring element of size $3 \times 3$ with $S_{i j}=1$. The choice of a limited set of pixels allows to improve the sensitivity of the cost function. 


\subsubsection{Description of the FE models}

The 2D FE geometry was either derived from the known geometry of the phantom or segmented from PC images. The contours of the artery were extracted in order to separate the arterial wall from the surrounding tissue and then separately estimate the elastic moduli of each. Quadratic triangular elements were used for the mesh generation (we previously showed that this type of elements provides accurate displacement fields for this type of problems [37]). A uniform pressure was applied to the phantom or artery inner walls. The implicit solver of Abaqus $($ C [38] was used.

Phantom A 2D cross section of the phantom was modeled. It was made up of 1100 finite elements surrounded by approximately 5000 similar elements mimicking the fluid.

The boundary conditions were enforced in the experiment. A maximal pressure of $9.33 \mathrm{kPa}(70 \mathrm{mmHg})$ was applied on the phantom's inner wall. The free edges of the surrounding box were fixed in the FE model (see Fig. 4).

Linear elastic material properties were used for the phantom. The Poisson's ratio was set to 0.49 while the elastic modulus has to be recovered. The external water had no mechanical influence because the external pressure applied on the phantom was constant during the test. It was nonetheless incorporated into the model in order to provide the necessary external displacement fields. Linear elasticity was considered with a Poisson's ratio of 0 and an elastic modulus of $10^{-6} \mathrm{kPa}$.

Subjects The FE geometry of each subject (see Fig. 6) was derived from the PC MR images. The contours of the neck, trachea, vertebral column and jugular vein were segmented manually from the global undeformed image (see Fig. 5a). The inner wall of the artery was recovered automatically using an algorithm based on watershed and Fourier polynomial descriptors: (i) gradients of the mean magnitude along the cardiac cycle were calculated using finite differences; (ii) watershed sources were chosen in the artery's lumen, in the vein's lumen and in the surrounding tissue; (iii) "contour pixels" were then deduced; (iv) the final contour was the approximation of these pixels' centers with a first degree Fourier descriptor. The thickness of the artery was measured by echography: the IMT (Intima-Media Thickness) was $e_{A}=0.45 \mathrm{~mm}$ 


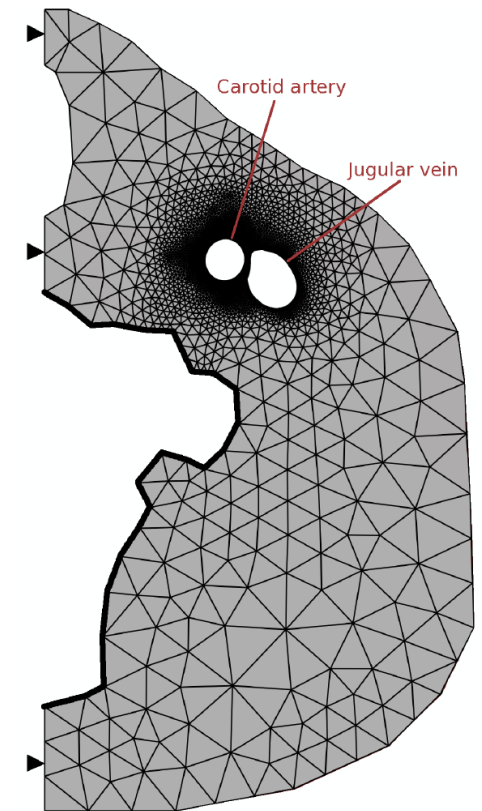

(a) Subject A (right common carotid).

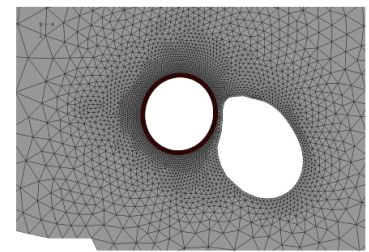

(c) Zoom on the carotid artery

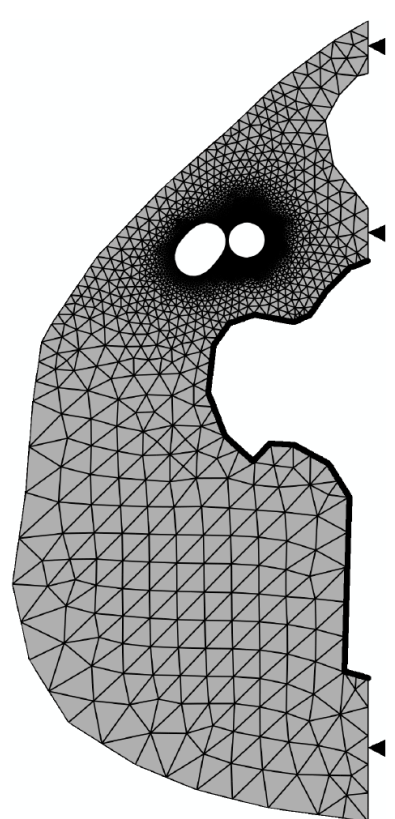

(b) Subject B (left common carotid).

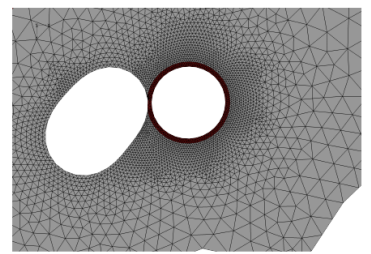

(d) Zoom on the carotid artery

Figure 6: FE meshes were made of triangular quadratic plane strain elements. The arteries were composed of approximately 1000 elements while the surrounding tissues were composed of approximately 7000 elements. A uniform pressure was applied to the inner lumen of the carotid artery. Vertical symmetry was assumed. Vertebral column was fixed (see bold black lines).

and $e_{B}=0.43 \mathrm{~mm}$ for each subject respectively. The outer contour of the artery was deduced from the inner contour and from the IMT.

The artery was represented using approximately 1000 elements and the surrounding tissue with about 7000 elements. Both the artery and the surrounding tissue were assumed linear elastic in the range of loading. Their Poisson's ratio was set to 0.49 as arteries were quasi incompressible. The elastic 
modulus of the artery was unknown and had to be identified while the elastic modulus of the surrounding tissue was set to $30 \mathrm{kPa}$. The influence of this modulus on the identified elastic modulus of the artery is studied herein (see Section "Results").

Regarding the boundary conditions, sagittal symmetry conditions were imposed for each model (horizontal symmetry). The vertebral column was assumed undeformable and the corresponding contour was fixed. Other contours were left free. The mean measured pulse pressure (differential pressure between diastolic and systolic states), measured before and after the exam with a digital sphygmomanometer, was applied to the artery's lumen $\left(\Delta P_{A}=\right.$ $11.33 \pm 0.13 \mathrm{kPa}$ with $P_{A}^{\text {diastole }}=72 \mathrm{mmHg}$ and $P_{A}^{\text {systole }}=157 \mathrm{mmHg}$, and $\Delta P_{B}=6.67 \pm 0.13 \mathrm{kPa}$ with $P_{B}^{\text {diastole }}=84 \mathrm{mmHg}$ and $P_{B}^{\text {systole }}=134 \mathrm{mmHg}$ ). As a first approximation, we assumed that the blood pressure in the carotid was equal to the blood pressure in the brachial artery. The values of these pulse pressures were discussed below. The reference state was set to the diastolic state.

\subsubsection{Definition of the parametric study}

A parametric study was carried out to evaluate the sensitivity of the described methodology on its internal parameters. The uniqueness of the solution was verified by changing the initial elastic modulus. The influences of (i) the choice of control pixels, (ii) potential errors on the estimation of physical parameters (mechanical properties of the surrounded tissues or measured pressure) and (iii) potential errors on the geometry of the artery were studied. The reference configuration is described in Table 2.

\section{Results}

In this section we focus on the final identified elastic moduli and on the values of the cost function at the optima. Results are given as mean \pm standard deviation. A full identification procedure requires approximately $5 \mathrm{~min}(8$ LevenbergMarquardt iterations). 
Table 2: Reference test configuration. Tests were conducted by modifying one parameter at a time.

\begin{tabular}{|c|c|c|c|}
\hline & Phantom & Subject A & Subject B \\
\hline Initial elastic modulus of the vessel & $300 \mathrm{kPa}$ & $500 \mathrm{kPa}$ & $500 \mathrm{kPa}$ \\
\hline Choice of control pixels & automatic $(N=704)$ & $\begin{array}{l}\text { automatic } \\
\left(N^{A}=130\right)\end{array}$ & $\begin{array}{l}\text { automatic } \\
\left(N^{B}=120\right)\end{array}$ \\
\hline Smoothing filter & $\begin{array}{l}\text { temporal Gaussian } \\
\quad \text { with } \sigma=1.5\end{array}$ & $\begin{array}{l}\text { temporal Gaussian } \\
\quad \text { with } \sigma=1.5\end{array}$ & $\begin{array}{l}\text { temporal Gaussian } \\
\quad \text { with } \sigma=1.5\end{array}$ \\
\hline Pressure applied & $9.33 \mathrm{kPa}$ & $11.33 \mathrm{kPa}$ & $6.666 \mathrm{kPa}$ \\
\hline $\begin{array}{l}\text { Elastic modulus of the surrounding tis- } \\
\text { sue }\end{array}$ & $0 \mathrm{kPa}$ & $30 \mathrm{kPa}$ & $30 \mathrm{kPa}$ \\
\hline Thickness of the vessel considered & $1.5 \mathrm{~mm}$ & $0.45 \mathrm{~mm}$ & $0.43 \mathrm{~mm}$ \\
\hline
\end{tabular}

\subsection{Validation of the MB-FEMU method with the phantom}

\subsubsection{Validation of the value of the identified properties}

A rubber phantom was used to validate the method. Its elastic properties in the range of loading were determined by an uni-axial tensile test (elastic modulus $E=250 \mathrm{kPa}$ and Poisson's ratio $v=0.49$ ). 32 tests combining different choices of control pixels, different initial elastic moduli and different smoothing parameters were conducted. The details of these results are presented herein. The mean identified elastic modulus is $E=274 \mathrm{kPa} \pm 19.8$ which corresponds to an average error $\varepsilon=9.6 \%$.

\subsubsection{Uniqueness of solution}

Our identification is based on solving the minimization problem (see Equation 4) with the Levenberg-Marquardt algorithm which is efficient for least squares formulations but which may get trapped at local minima of $J_{2}$. In order to study the uniqueness of the solution to the minimization problem, five different initial values of the elastic modulus between 150 and $350 \mathrm{kPa}$ have been input to the identification algorithm. Results are $E_{\text {mean }}=270 \mathrm{kPa} \pm 1.09$ and $J_{2}^{\text {mean }}=3.34 \cdot 10^{4} \pm 2.64$. Note that the standard deviations are very low which means that the identification is marginally affected by a change of the initial value of the elastic modulus. 


\subsubsection{Effect of the choice of control pixels}

The choice of the pixels considered in the cost function may have an influence on the identified elastic modulus. The control pixels can be either selected automatically using the procedure described previously or chosen manually by defining a binary mask which contains the vessel. In this section, 15 different binary masks were tested. For each one of them, a different number of control pixels was assigned (from 704 to 1320 control pixels). Results are $E_{\text {mean }}=280 \mathrm{kPa} \pm 4.93$ which means that the variations of the binary mask don't affect the identified modulus. Note nevertheless that the best identified elastic modulus occurs when the control pixels are chosen automatically (704 control pixels; $E=271 \mathrm{kPa}$ ). The value of the cost function at optimum tends to slightly decreases with the increase of the number of control pixels (results are $J_{2}^{\text {mean }}=2.67 \cdot 10^{4} \pm 0.23 \cdot 10^{4}$ ). However there is no correlation between this decrease of values and a change on the identified moduli.

\subsubsection{Effect of the smoothing filter}

We also studied the effect of filtering the data before the identification procedure (see Fig. 7a and $7 \mathrm{~b}$ ). Two types of filters were tested: temporal filters (Gaussian filter as described previously with various kernel sizes), and spatial filters (Wiener and median with different kernel sizes). The overall results accounting for both filters are $E_{\text {mean }}=268 \mathrm{kPa} \pm 31.09$ and $J_{2}^{\text {mean }}=3.27 \cdot 10^{4} \pm$ $0.88 \cdot 10^{4}$. The temporal filter tends to increase the estimated elastic modulus (Fig. 7a). The elastic modulus ranges from $E=265 \mathrm{kPa}$ to $E=341 \mathrm{kPa}$ while the value of the cost function decreases linearly with the increase of kernel size $\sigma$ (Fig. 7b). Median spatial filtering yields moduli close to the tensile test reference value (kernels $3 \times 3$ and $5 \times 5: E=256 \mathrm{kPa}$ and $E=254 \mathrm{kPa}$ ). Wiener spatial filtering underestimates the elastic modulus (kernels $3 \times 3$ and $5 \times 5$ : $E=231 \mathrm{kPa}$ and $E=217 \mathrm{kPa})$.

\subsubsection{Effect of measurement errors in the pressure}

Errors of plus or minus $15 \%$ were considered for the nominal pressure $P$ in the phantom (see Fig. 8a). Results show that the value of the cost function slightly decreases with the increase of pressure. Nevertheless, the standard deviation is very low which makes the cost function almost unaffected $\left(J_{2}^{\text {mean }}=\right.$ 


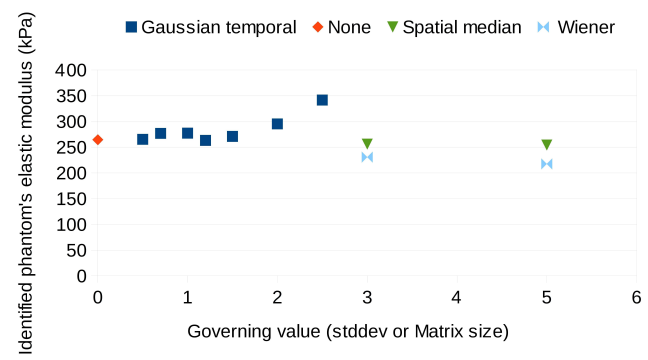

(a) Identified elastic modulus for the phantom.

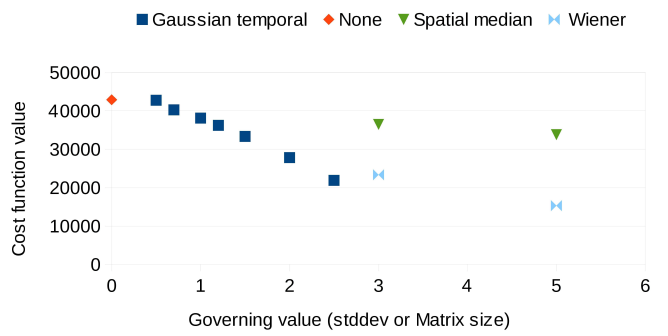

(b) Values of the cost function at the optima for the phantom

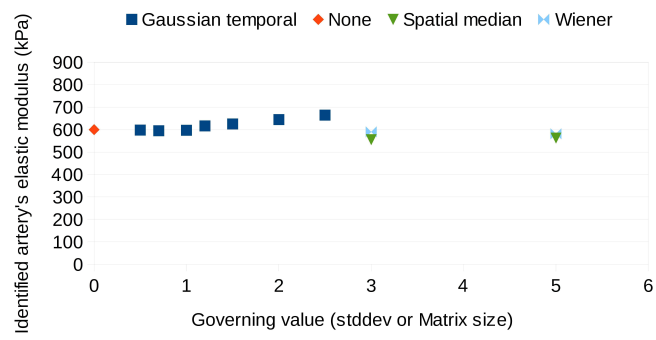

(c) Identified elastic modulus for subject A.

Figure 7: Effect of the raw images filtering. The filter was applied to both the initial and the deformed images. Final identified elastic moduli (squares, left axis) and the values of the cost function at the optima (diamonds, right axis) are shown for the phantom and the two subjects.

$\left.3.34 \cdot 10^{4} \pm 3.74\right)$. The identified elastic modulus ranges from $E_{\min }=231 \mathrm{kPa}$ to $E_{\max }=307 \mathrm{kPa}$ which corresponds to errors of $-7.6 \%$ to $+22.8 \%$. There is a clear linear relationship between the pressure and the identified modulus 


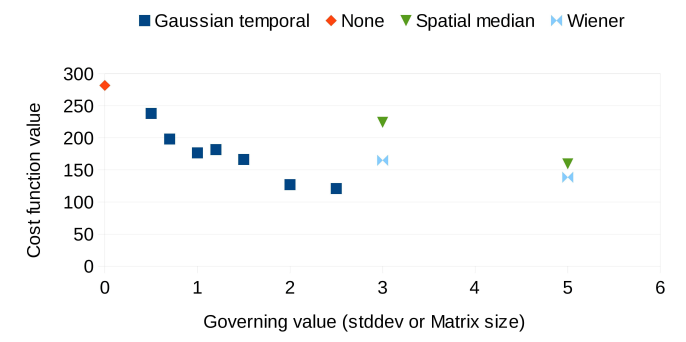

(d) Values of the cost function at the optima for subject A.

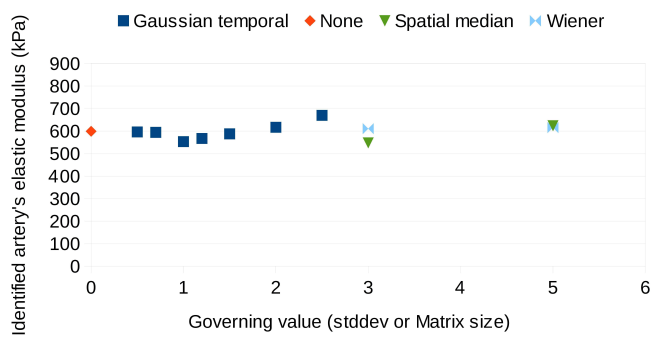

(e) Identified elastic modulus for subject B.

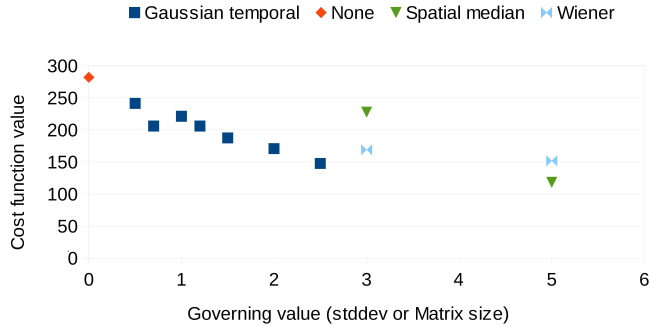

(f) Values of the cost function at the optima for subject B.

Figure 7: (continued with last three figures) 
$(r=0.99)$.

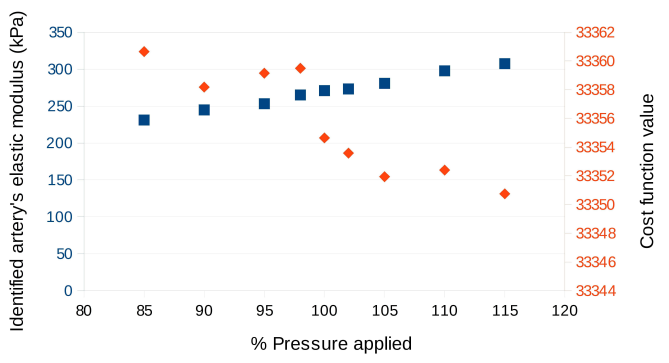

(a) Phantom.

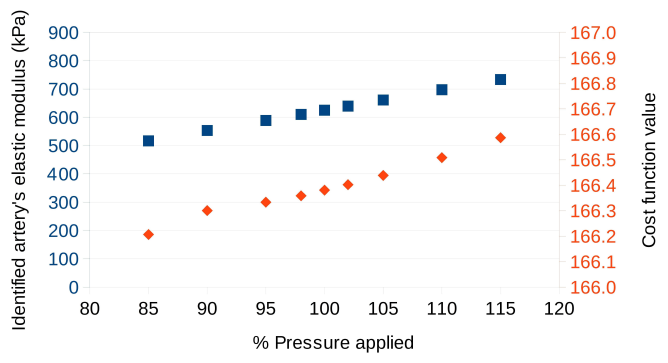

(b) Subject A.

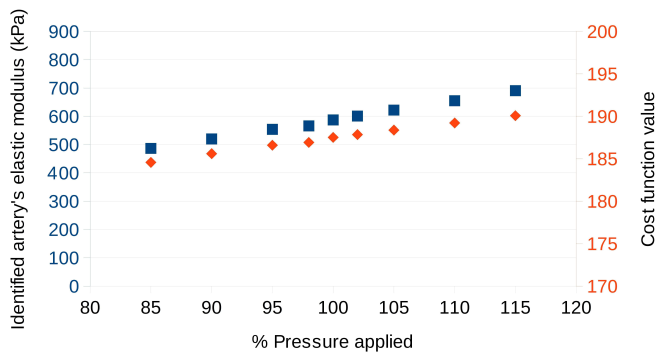

(c) Subject B.

Figure 8: Effect of the applied pressure in the FE model for the phantom and both subjects. Squares and diamonds correspond to the identified elastic moduli (left axis) and to the values of the cost function at the optima (right axis), respectively. 


\subsection{Healthy subjects study}

For the subjects, the same numerical experiments were conducted for the variations of the identification parameters and for the effect of the estimation of pressure on the identified modulus.

\subsubsection{Identification parameter variations}

Uniqueness of solution The initial elastic modulus of the artery was changed six times to study the uniqueness of the solution in the range 400 to $900 \mathrm{kPa}$. Results are $E_{\text {mean }}^{A}=625 \mathrm{kPa} \pm 0.27$ and $E_{\text {mean }}^{B}=586 \mathrm{kPa} \pm 3.25$. The value of the cost function at the optimum is unaffected: $J_{2 A}^{m e a n}=166.38 \pm 5.10^{-4}$ and $J_{2 B}^{\text {mean }}=187.48 \pm 0.10$. As well as the results on the phantom, the identified elastic modulus is independent of the initial elastic modulus.

Effect of the choice of control pixels The choice of different sets of control pixels doesn't affect the identified elastic modulus (results are $E_{\text {mean }}^{A}=$ $630 \mathrm{kPa} \pm 13.50$ and $E_{\text {mean }}^{B}=589 \mathrm{kPa} \pm 9.66$ for 15 sets of control pixels which contain from 101/89 to 220/167 pixels for A/B, respectively). In each case the identified elastic moduli are similar while the values of the cost function decrease with the increase of the number of control pixels. This observation is similar to what we found with the phantom.

Effect of the smoothing filter Results are summarized in Fig. 7c, 7d, 7e and $7 \mathrm{f}$. The average results are $E_{\text {mean }}^{A}=602 \mathrm{kPa} \pm 31.45$ and $E_{\text {mean }}^{B}=598 \mathrm{kPa} \pm$ 33.38 . The identified moduli range from $555 \mathrm{kPa}$ to $664 \mathrm{kPa}$ for subject $\mathrm{A}$ and from $547 \mathrm{kPa}$ to $670 \mathrm{kPa}$ for subject $\mathrm{B}$. The minimum and maximum values were obtained in both cases for the $3 \times 3$ median filter and for the 2.5 Gaussian filter, respectively. The value of the cost function at optimum decreases with the augmentation of the "strength" of the filtering although the comparison between the temporal and the spatial filters is not easy. However the filtering of images always reduces the value of the cost function at optimum compared to using the raw images. 


\subsubsection{Effect of the estimation of physical parameters}

Effect of measurement errors in the pressure Pulse blood pressure (difference between maximal and minimal pressures) was measured with a digital sphygmomanometer $\left(\Delta P_{A}=11.33 \mathrm{kPa}\right.$ and $\left.\Delta P_{B}=6.67 \mathrm{kPa}\right)$. Similarly to the phantom validation, a variation of plus or minus $15 \%$ were considered for the nominal pressure $\Delta P$ applied in the FE model. Results are detailed in Fig. $8 \mathrm{~b}$ and 8c. Identified elastic moduli range from $E_{\text {min }}^{A}=517 \mathrm{kPa}$ to $E_{\text {max }}^{A}=734 \mathrm{kPa}$ for subject $\mathrm{A}$ and from $E_{\text {min }}^{B}=487 \mathrm{kPa}$ to $E_{\text {max }}^{B}=691 \mathrm{kPa}$ for subject $\mathrm{B}$. The relationship between the pressure and the identified modulus is linear which confirms the results from the phantom. Nevertheless and contrary to the phantom study, the cost function value at optimum tends to increase linearly with a higher pressure in both subjects.

Effect of the estimation of the mechanical properties of the surrounding tissue The artery was assumed to be surrounded by a soft elastic media. Its elastic properties were supposed to be known. The choice of its elastic properties may have an influence on the identification of the artery's stiffness. For instance, in Fig. 9, we show how the choice of the elastic modulus of the surrounding tissue affects the identification of the elastic modulus of the artery. As the surrounding tissue stiffness is fixed to increasingly higher values, the identified elastic modulus of the artery decreases. The values of the cost function exhibits a second order polynomial shape with a minimum reached for $E_{\text {surrounding }}^{A} \approx 30 \mathrm{kPa}$ and $E_{\text {surrounding }}^{B} \approx 150 \mathrm{kPa}$. This shows that the cost function is sensitive to the stiffness of the surrounding tissue. The modification of the elasticity of the surrounding tissue corresponds somehow to a modification of the pressure applied at the outer boundary of the artery. Previous results on the effect of the pressure shows a linear relationship between the identified moduli or the value of the cost function at optimum and the inner pressure applied. Here the correlation between the elastic modulus of the surrounding tissue and the identified artery's modulus is linear in both cases $\left(r^{A}=0.98\right.$ and $\left.r^{B}=0.94\right)$. For the cost function however, the relationship with the elastic properties of the surrounding tissue seems to be a quadratic form. 


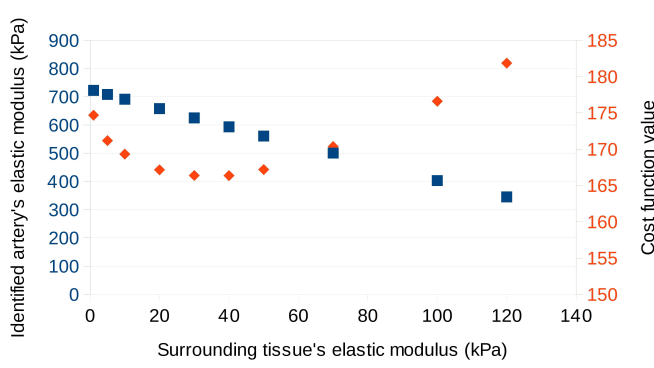

(a) Subject A.

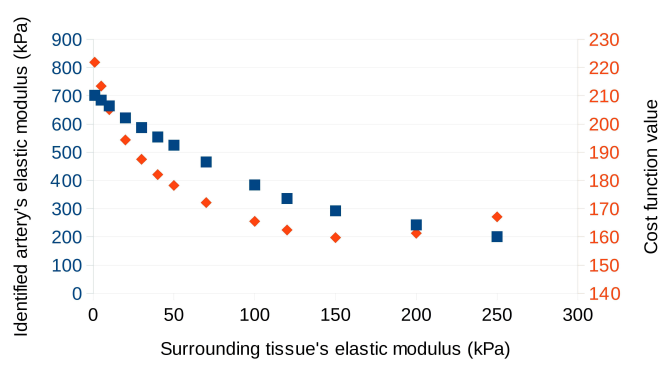

(b) Subject B.

Figure 9: Influence of the elastic modulus of the surrounding tissue on the identified elastic modulus of the artery (squares, left axis) and on the value of the cost function value at the optima (diamonds, right axis).

\subsubsection{Effects of errors in the geometry}

Errors in the artery's position The FE model geometry was defined from the undeformed image. Potential errors in the positioning of the artery were considered (see Fig. 10). No tendency appears with the X-axis or Y-axis offsets neither for the identified moduli nor for the values of the cost function at the optima.

\section{Effect of measurement errors in the artery's diameter The inner lumen of the} artery is determined automatically (see section "Methodology"). This section aims at studying the effect of a wrong estimation of the inner diameter due to the algorithm described previously. Errors of plus or minus $10 \%$ of the nominal diameter were considered (see Fig. 11). For subject B, the diameter of the artery could not be increased further because it was already in contact 


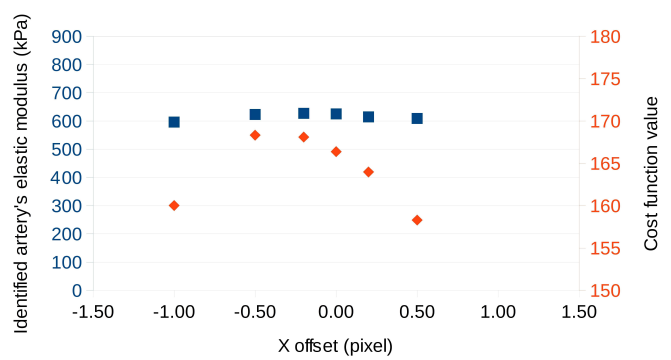

(a) Error of the position of the artery along the $\mathrm{X}$-axis for subject $\mathrm{A}$.

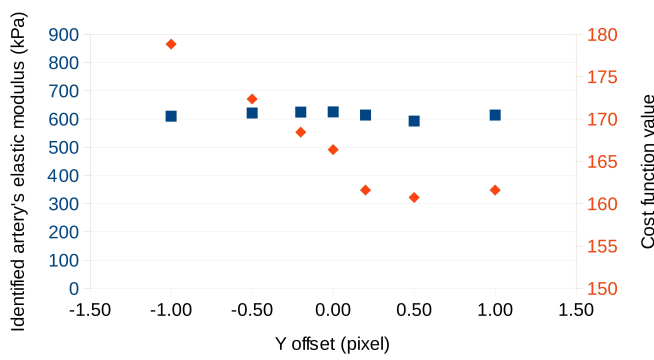

(b) Error of the position of the artery along the Y-axis for subject A.

Figure 10: Study of potential errors in the positioning of the artery in the FE model. Squares represent the final identified elastic moduli (left axis) and diamonds the values of the cost function at the optima (right axis).

with the jugular vein. The resulting elastic moduli identified lie within the following ranges respectively for subjects $\mathrm{A}$ and $\mathrm{B}: E_{\text {range }}^{A}=[553 \mathrm{kPa}, 659 \mathrm{kPa}]$ and $E_{\text {range }}^{B}=[487 \mathrm{kPa}, 587 \mathrm{kPa}]$. The identified moduli increase with diameter. The value of the cost function at the optimum decreases when the diameter is reduced.

Effect of measurement errors in the artery's thickness The thickness of the artery was measured with ultrasounds. Errors of plus or minus $10 \%$ in the thickness of the artery were considered (see Fig. 12). The elastic moduli ranges resulting from the identification process are respectively $E_{\text {range }}^{A}=[566 \mathrm{kPa}, 696 \mathrm{kPa}]$ and $E_{\text {range }}^{B}=[533 \mathrm{kPa}, 660 \mathrm{kPa}]$. The correlation between the identified modulus and the thickness of the artery is linear, with a decrease of the identified 


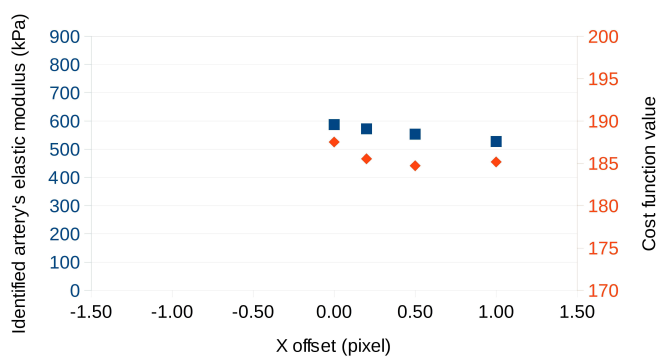

(c) Error of the position of the artery along the $\mathrm{X}$-axis for subject $B$.

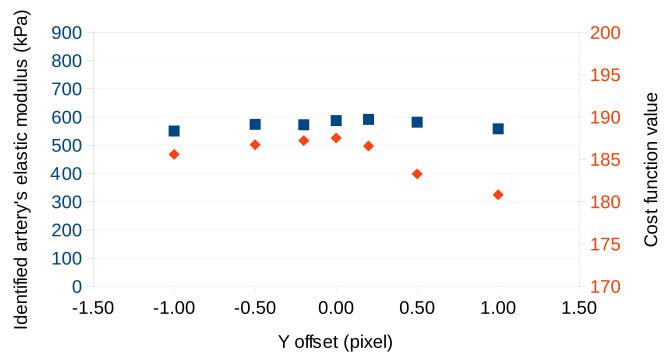

(d) Error of the position of the artery along the Y-axis for subject $B$.

Figure 10: (continued with last two figures)

484 low.

\section{${ }_{485} 4$ Discussion}

486 The discussion is organized in two parts. The first part focuses on the robust${ }_{487}$ ness of the MB-FEMU method whereas the second part discusses the elastic ${ }_{488}$ properties of arteries obtained.

\section{${ }_{489}$ 4.1 Robustness of the MB-FEMU method}

490 In the three cases studied in the present work (the phantom and the two sub-

${ }_{491}$ jects) the robustness of the MB-FEMU method was tested with respect to dif- 


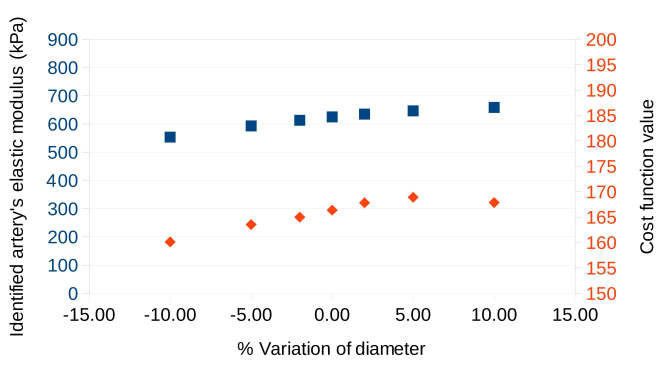

(a) Subject A.

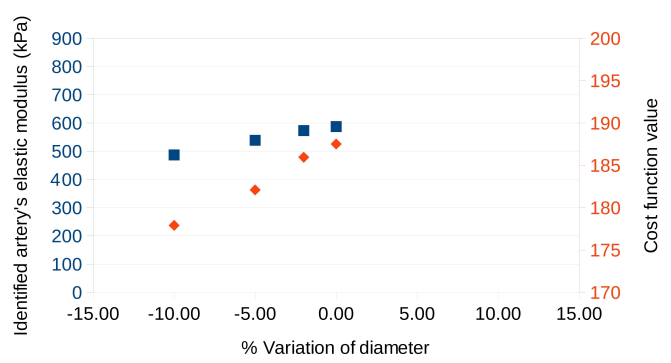

(b) Subject B.

Figure 11: Effect of the measurement error of the artery's diameter. Squares represents the elastic moduli (left axis) and diamonds the values of the cost function at the optima (right axis).

ferent sources of uncertainty and artifacts. The normalized input parameters were noted $X_{i}$. Assuming that the relationship between the identified elastic modulus and parameter $X_{i}$ is linear, it is possible to rank the influence of each parameter by calculating the following normalized regression coefficient:

$$
a_{i}=\left|\frac{\operatorname{Cov}\left(X_{i}, E\right)}{\operatorname{Var}\left(X_{i}\right)}\right|
$$

where $E$ is the identified elastic modulus and $X_{i}$ is the normalized parameter $i\left(X_{i} \in[0,1]\right)$. The higher the coefficient $a_{i}$, the higher the influence of the parameter on the identified variable. Results are shown in Table 3.

The sources of uncertainty and artifacts can be sorted into three groups according to their relative influence:

1. The most important parameter is the pressure applied on the vein wall (i) by the surrounding soft tissue on the outer surface, (ii) and by the 


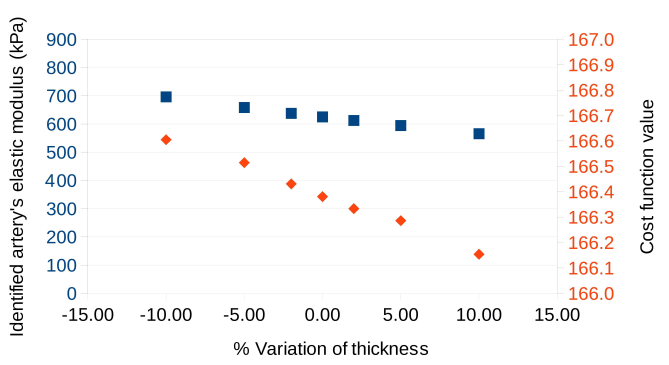

(a) Subject A.

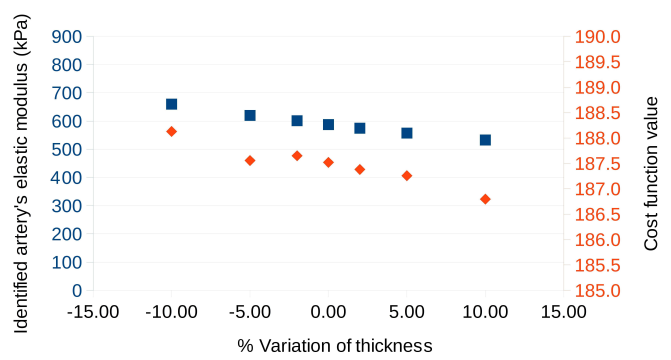

(b) Subject B.

Figure 12: Effect of the measurement error of the artery's thickness. External diameter remains constant in all cases and only the internal diameter was modified. The identified elastic moduli (left axis) and the values of the cost function at the optima (right axis) are represented as squares and diamonds, respectively.

blood circulation on the inner surface. It is particularly obvious for subject $B$ (see Fig. 9b). The estimation of the stiffness of the surrounding tissue and of the pressure applied is crucial to accurately identify the elastic modulus of the artery. It can be observed in Fig. 9a and 9b that the value of the cost function evolves when the stiffness of the surrounding tissue is increased in the model. A minimum is reached for the values $E_{\text {surrounding }}^{A} \approx 30 \mathrm{kPa}$ and $E_{\text {surrounding }}^{B} \approx 150 \mathrm{kPa}$. This result suggests that the identification process can be used to determine the elastic moduli of both the artery and the surrounding tissue simultaneously. In this case the uniqueness of the solution would not be guaranteed and the computation time would increase. The asymmetric displacement of the artery between the configurations (see Fig. 13) suggests the presence of a stiffer 
tissue on the right hand side of the artery (tendons for instance).

2. Less important but not negligible are the sources of uncertainty that may arise from the geometry definition. On one hand, we observe that the error made on the artery position does not significantly significantly affect the identified elastic modulus. This constitutes one of the strength of the MB-FEMU methodology. On the other hand, the diameter and the thickness have a moderate but significant influence and thus, must be characterized accurately. It is possible to define a parametric model as in [30] to recover simultaneously both the elastic modulus and the geometry of the artery (in this case the uniqueness of the solution would not be guaranteed and the computation time would probably increase). Note that the entire thickness of the artery is not taken into account here (only the Intima-Media Thickness is considered) but that extra information on the total thickness could help reduce this uncertainty as the total wall thickness can be measured by ultrasounds or MRI with a Turbo Spin Echo flash sequence (Blackblood-FATSAT) [39].

3. The least important sources of uncertainty results from the so-called identification parameters: (i) position and number of control pixels, (ii) initial elastic modulus. The induced errors can therefore be neglected as compared to those induced by the other sources. Identification results (very close one to the other) obtained from different sets of initial parameters are a good hint that the solution of the problem is very likely to be unique. Only the kernel sizes of the filters must be chosen with care because an increase of the kernel size decreases the apparent deformation of the images between the initial and deformed configurations (see Fig. 7).

\subsection{Identified elastic moduli}

Arterial compliance has been widely studied over the last decades. Stiffness values found in the literature are widespread for two main reasons: (i) subjects doing the experiment are different (important inter-individual differences), (ii) methods are different. The review of [40] on the calculation of arterial stiffness indexes in clinical practice mentioned nine different stiffness indexes from the "elastic modulus" to the "stiffness index". Recently [41] listed existing meth- 


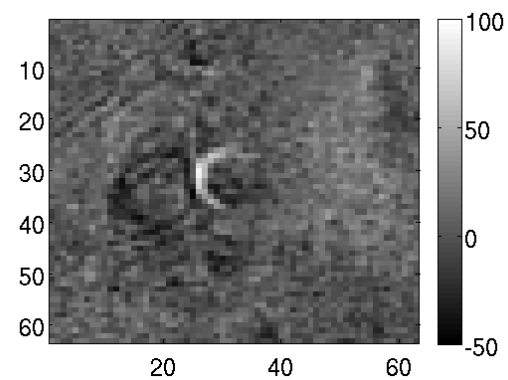

Figure 13: Difference between experimental deformed and initial images for subject B. A white crown appears around the artery. The asymmetry of displacement of the artery can be due to the high stiffness of tissues at the right hand side (tendons, etc.).

Table 3: Normalized regression coefficients $a_{i}$, measuring the influence of the different parameters on the identified elastic modulus of the artery, are calculated with Equation (10).

\begin{tabular}{ccc}
\hline & Subject A & Subject B \\
\hline E surrounding tissue & 339.87 & 487.76 \\
Pressure & 192.64 & 181.41 \\
Thickness & 111.31 & 108.76 \\
Diameter & 90.64 & 76.96 \\
Control pixels & 43.61 & 26.40 \\
Translation dX & 11.85 & 44.48 \\
Translation dY & 8.40 & 10.18 \\
Initial elastic modulus & 0.39 & 2.84 \\
\hline
\end{tabular}

ods which are used in clinical practice to assess arterial stiffness. The same author defined in [16] the incremental modulus of an artery such as:

$$
E_{i n c}=\left(3\left(1+\frac{R_{d}^{2}}{\left(R_{d}+I M T\right)^{2}-R_{d}^{2}}\right)\right) \cdot \frac{R_{d}^{2} \cdot \Delta P}{R_{s}^{2}-R_{d}^{2}}
$$

where $R_{S}$ and $R_{d}$ are respectively the systolic (highest pressure) and diastolic (lowest pressure) radii of the artery; IMT is the Intima-Media Thickness; $\Delta P$ is the pulse pressure between diastole and systole. This formula was determined for an isotropic, incompressible, semi closed thick cylinder with an inner pressure applied. 


\subsubsection{Comparison with ultrasounds measurements}

The MB-FEMU method presented in this paper yields elastic moduli of $625 \mathrm{kPa}$ and $587 \mathrm{kPa}$ when applied to two different young healthy subjects. In addition an echography was conducted on these subjects in order to acquire movies of their carotid arteries during three heart beats. Initial and deformed images were extracted manually for both subjects (see Fig. 14). Pulse blood pressures were measured during the exam with a digital sphygmomanometer: $\Delta P^{A}=8.27 \mathrm{kPa}$ and $\Delta P^{B}=6.40 \mathrm{kPa}$. Arteries lumens were segmented manually on the initial and deformed ultrasounds images and the corresponding incremental modulus was calculated with Equation (11). The implementation of the MB-FEMU method was based on the assumption of plane strain in a cross section of the artery which is different from the assumptions used for deriving Equation (11). In order to make some equivalence between the methods, initial and deformed areas (and subsequently radii) were segmented directly from the results of the FE computation at the optima for calculating $E_{\text {inc }}$ using Equation (11). The pressures used in the case of the FE model were $\left\{\Delta P_{A}, \Delta P_{B}\right\}=\{11.33 \mathrm{kPa}, 6.666 \mathrm{kPa}\}$. Results are $E_{\text {inc }}^{\text {echo }}=374 \mathrm{kPa} ; E_{\text {inc }}^{F E}=$ $496 \mathrm{kPa}$ and $E_{\text {inc }}^{\text {echo }}=434 \mathrm{kPa} ; E_{\text {inc }}^{F E}=509 \mathrm{kPa}$ for the subjects $\mathrm{A}$ and $\mathrm{B}$, respectively.

It can be noted that the results obtained with both imaging systems are consistent with the values reported in the literature (see below) though small differences can be noted. The differences between the two imaging systems can be justified by three main reasons. First, the boundary conditions enforced in each model are different because the ultrasound probe applied a pressure on the neck during the exam. Secondly, the height on the neck at which the ultrasound images were taken is not managed and is potentially different from the height of the MRI. Third, the blood pressure of the patients were sensibly different during the MRI and the echography exams $\left(\Delta P_{A}^{\text {echo }}=8.27 \mathrm{kPa}\right.$ vs $\left.\Delta P_{A}^{M R I}=11.33 \mathrm{kPa} ; \Delta P_{B}^{e c h o}=6.40 \mathrm{kPa} v s \Delta P_{B}^{M R I}=6.666 \mathrm{kPa}\right)$. This potentially could impact the behavior of the arterial wall which is non-linear. Differences in pulse pressures between MRI and echography as well as higher pulse pressure in MRI were observed several times for some subjects. It can be explained by some psychological stress induced by the noisy and confined environment. 


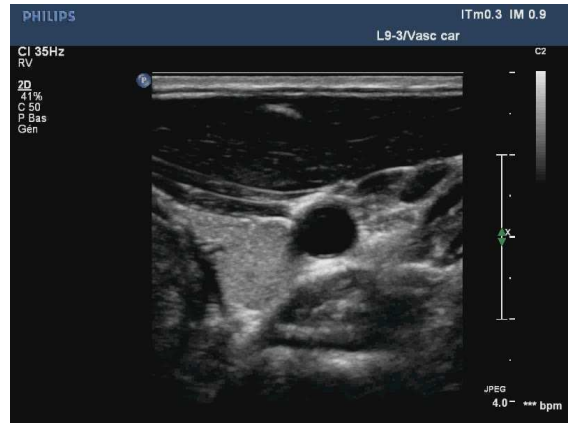

(a) Initial image for subject A.

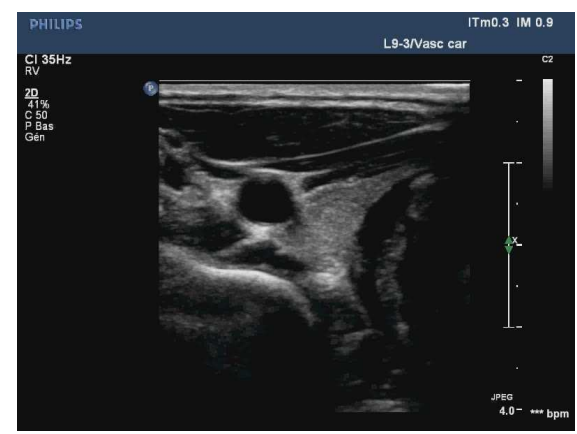

(c) Initial image for subject B.

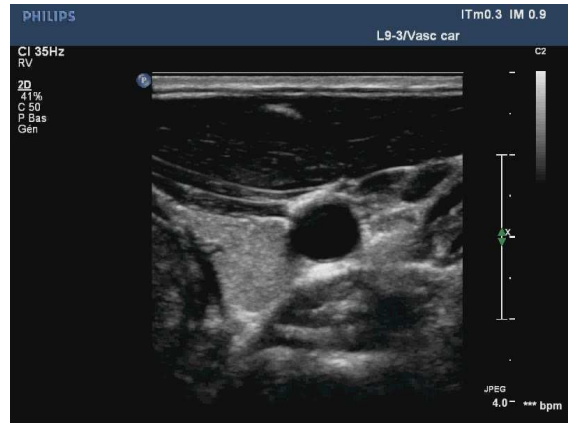

(b) Deformed image for subject A.

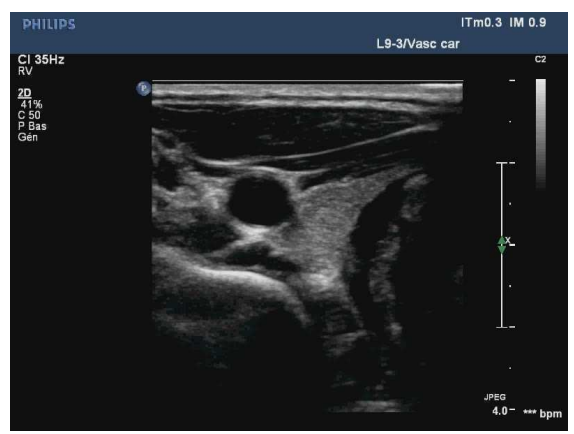

(d) Deformed image for subject B.

Figure 14: Ultrasounds image (echography) of the carotid arteries of both subjects. Initial (diastole) and deformed (systole) images were extracted manually from movies taken during three heart beats.

Artery's stiffness A survey of different in vivo elastic moduli identified by other authors is reported in Table 1. Different imaging systems were used: Bmode echography (see Fig. 14), Radio-Frequency UltraSounds signal, IntraVascular UltraSounds or MRI. The reported elastic moduli are in good agreement with our results in most of the studies except for: (i) [16] ( $E=2680 \pm 1810 \mathrm{kPa})$ where the difference may be explained by the location of the artery (radial artery at the wrist instead of carotid artery); (ii) [25] ( $E=1400 \mathrm{kPa})$ where the elastic modulus depends on the excitation frequency of the ultrasounds because a viscoelastic behavior is considered; (iii) [42] ( $E=188 \mathrm{kPa})$ where a different artery was also studied (pathological coronary artery); (iv) [18] ( $E_{\text {inc }}=$ 
$187 \pm 67 \mathrm{kPa}$ ) where the subjects were significantly younger (9-year-old); (v) [13] $(E=99 \mathrm{kPa})$ where the authors suggest that the small elastic modulus found may be related to a bad estimation of the thickness.

MB-FEMU method vs other methods The methods used in the literature for quantifying the artery's stiffness in vivo are generally based on the variation of the artery's diameter during heart beats. In these cases authors generally used theoretical formula by considering several mechanical and geometrical assumptions: $[16,17,18,19,20,24]$ calculated the incremental modulus according to Equation (11); $[15,21]$ applied the Laplace law by considering a 2D plane stress artery; $[22,25]$ derived an analytical formula of the circumferential elastic modulus using the assumptions that the artery is incompressible, isotropic and that the arterial pressure decreases linearly from the artery's inner diameter to adventitia). Other studies derived the artery's stiffness from velocity Phase Contrast MR images and Moens-Korteweg equation which relates the stiffness of a round artery to the pulse wave velocity and a round artery's stiffness [13] or studied the propagation of shear waves induced by an ultrasonic device to deduce shear modulus (denoted $\mu$ ) and the elastic modulus (denoted $E)$ according to $E=3 \cdot \mu[14]$.

Few studies measured the stiffness of the human carotid arteries by taking into account the surrounding tissue and the environment of the artery (vertebral column, tendons, etc.). [42] used a strain-based FEMU method to recover both the contours and the elastic moduli of the constituents of an atherosclerotic plaque. This method is very invasive since the IVUS technique was employed to obtain strain fields in the wall of the artery. [43] used another FEMU method with a cost function defined as a difference of areas but the areas were segmented manually from Phase Contrast images which may be critical with regard to the pixel size.

Our method and these alternative FEMU methods have in common the advantage that they are not based on strong mechanical assumptions. Indeed the arteries are known to behave highly non-linearly $([5,44,45,12])$. Only the tangent (or incremental) behavior is considered for the moment by linearizing the stress/strain relationship of arteries between diastole and systole. The identified elastic modulus is therefore a function of the diastolic and pulse pressures due to the intrinsic non-linear behavior of arteries. More complex constitu- 
tive equations could potentially be identified by including into the FE model anisotropy, viscoelastic effects, heterogeneity, etc. The limit is the question of the identifiability and uniqueness of the solution that should be investigated for each other constitutive equations. Compared to alternative FEMU methods reported in the literature, the MB-FEMU method has also the advantage of being compatible with non-invasive imaging techniques such as MRI. Indeed, clinical MRI was used and the impact on the patient is very limited (standard and routinely-used exam for a patient before carotid endarterectomy).

Close to our method, the "hyper elastic warping" is a technique that was developed a few years ago $([46,47,48])$. The objective is to perform image registration in order to quantify the kinematics of the transformation within a deforming body. Our approach is similar in the sense that we perform image registration as well, with the difference that our main objective is not to quantify the kinematics of the deformations but to identify the constitutive properties of the deforming body.

\section{Conclusions}

This study shows that identifying elastic moduli in common carotid arteries in vivo from cine-MRI images is possible. An original method named the Magnitude Based Finite Element Model Updating (MB-FEMU) methodology has been thoroughly described, validated on a rubber phantom and applied to two healthy subjects. In our implementation MRI and Phase Contrast magnitude images were used to provide the experimental data. The phase information of the Phase Contrast sequence could be used for improving the boundary conditions in the FE model as a perspective. We would like to bring the reader's attention to the fact that the MB-FEMU can be easily extended to other imaging techniques. The identified elastic moduli on healthy subjects are in good agreement with those found in the literature, especially if we consider the differences in hypotheses made that we discussed in the article. The MB-FEMU method offers several advantages compared to existing methods: it makes a maximum use of experimental data since the FE results are used only as a perturbation of the experimental acquisitions; it is possible to extract arterial stiffness from a routine MRI exam on any patient; it can readily be extended to more complex tissue constitutive behaviors. Heterogeneous geometries can be possibly iden- 
tified if an accurate FE model can be created, although the uniqueness of the minimization problem will have to be verified. In the future this method will be employed to study the modification of the mechanical behavior of arteries with age and cardiovascular disease. Applications for the diagnosis of vulnerable atherosclerotic plaques in the carotid is probably the most promising challenge for this method.

\section{Acknowledgment}

We are grateful to the two volunteers for their time and implication.

We would like to thank IEEE Transactions on Medical Imaging (please visit http://ieeexplore.ieee.org/xpl/RecentIssue.jsp?punumber=42)

\section{References}

[1] B. A. Kingwell, T. K. Wadell, T. L. Medley, J. D. Cameron, and A. M. Dart, "Large artery stiffness predicts ischemic threshold in patients with coronary artery disease," Journal of the American College of Cardiology, vol. 40, no. 4, pp. 773-779, 2002.

[2] R. T. Lee, A. J. Grodzinsky, E. H. Frank, R. D. Kamm, and F. J. Schoen, "Structure-dependent dynamic mechanical behavior of fibrous caps from human atherosclerotic plaques," Circulation, vol. 83, no. 5, pp. 1764-1770, 1991.

[3] A. Duprey, K. Khanafer, M. Schlicht, S. Avril, D. Williams, and R. Berguer, "In vitro characterisation of physiological and maximum elastic modulus of ascending thoracic aortic aneurysms using uniaxial tensile testing," European Journal of Vascular and Endovascular Surgery, vol. 39, no. 6, pp. 700-707, 2010.

[4] D. Beattie, C. Xu, R. Vito, S. Glagov, and M. C. Whang, "Mechanical analysis of heterogeneous, atherosclerotic human aorta," Journal of Biomechanical Engineering, vol. 120, no. 5, pp. 602-607, 1998.

[5] Y. C. Fung, Biomechanics : Mechanical properties of living tissues, 2nd ed. New-York: Springer-Verlag, 1993. 
[6] G. A. Holzapfel, G. Sommer, and P. Regitnig, "Anisotropic Mechanical Properties of Tissue Components in Human Atherosclerotic Plaques," Journal of Biomechanical Engineering, vol. 126, no. 5, pp. 657-665, 2004.

[7] C. L. Lendon, M. J. Davies, P. D. Richardson, and G. V. R. Born, “Testing of small connective tissue specimens for the determination of the mechanical behaviour of atherosclerotic plaques," Journal of Biomechanical Engineering, vol. 15, pp. 27-33, 1993.

[8] H. M. Loree, B. J. Tobias, L. J. Gibson, R. D. Kamm, D. M. Small, and R. T. Lee, "Mechanical properties of model atherosclerotic lesion lipid pools," Arteriosclerosis Thrombosis and Vascular Bioology, vol. 14, pp. 230-234, 1994.

[9] S. R. H. Barrett, M. P. F. Sutcliffe, S. Howarth, Z.-Y. Li, and J. H. Gillard, "Experimental measurement of the mechanical properties of carotid atherothrombotic plaque fibrous cap," Journal of Biomechanics, vol. 42, pp. 1650-1655, 2009.

[10] J. O. V. Delgadillo, "Mechanical properties of arterial wall," Ph.D. dissertation, University of British Columbia, 2008.

[11] G. A. Holzapfel, "Biomechanics of Soft Tissues with Application to Arterial Walls," in Mathematical and Computational Modeling of Biological Systems, J. A. C. Martins and E. A. C. Borges Pires, Eds. Coimbra, Portugal: Centro Internacional de Matemática CIM, 2002, pp. 1-37.

[12] E. Peña, V. Alastrué, A. Laborda, M. A. Martínez, and M. Doblaré, “A constitutive formulation of vascular tissue mechanics including viscoelasticity and softening behaviour," Journal of Biomechanics, vol. 43, no. 5, pp. 984-989, 2010.

[13] S. Avril, J. M. Huntley, and R. Cusack, "In-vivo measurements of blood viscosity and wall stiffness in the carotid using PC-MRI," European Journal of Computational Mechanics, vol. 18, pp. 9-20, 2009.

[14] M. Couade, M. Pernot, C. Prada, E. Messas, J. Emmerich, P. Bruneval, A. Criton, M. Fink, and M. Tanter, "Quantitative assessment of arterial wall biomechanical properties using shear wave imaging," Ultrasound in Medicine E Biology, vol. 36, no. 10, pp. 1662-1676, 2010. 
[15] W. A. Riley, R. W. Barnes, G. W. Evans, and G. L. Burke, “Ultrasonic Measurement of the Elastic Modulus of the Common Carotid Artery," Stroke, vol. 23, no. 7, pp. 952-956, 1992.

[16] S. Laurent, X. Girerd, J. J. Mourad, P. Lacolley, L. Beck, P. Boutouyrie, J. P. Mignot, and M. Safar, "Elastic modulus of the radial artery wall material is not increased in patients with essential hypertension," Arteriosclerosis and Thrombosis, vol. 14, no. 7, pp. 1223-1231, 1994.

[17] P. J. Brands, A. P. G. Hoeks, J. Willigers, C. Willekes, and R. S. Reneman, "An integrated system for the non-invasive assessment of vessel wall and hemodynamic properties of large arteries by means of ultrasound," European Journal of Ultrasounds, vol. 9, pp. 257-266, 1999.

[18] Y. Aggoun, D. Sidi, B. I. Levy, S. Lyonnet, J. Kachaner, and D. Bonnet, "Mechanical properties of the common carotid artery in Williams syndrome," Heart, vol. 84, pp. 290-293, 2000.

[19] C. Bussy, P. Boutouyrie, P. Lacolley, P. Challande, and S. Laurent, "Intrinsic stiffness of the carotid arterial wall material in essential hypertensives," Hypertension, vol. 35, no. 5, pp. 1049-1054, 2000.

[20] P. Boutouyrie, D. P. Germain, A.-I. Tropeano, B. Laloux, F. Carenzi, M. Zidi, X. Jeunemaitre, and S. Laurent, "Compressibility of the Carotid Artery in Patients With Pseudoxanthoma Elasticum," Hypertension, vol. 38, no. 5, pp. 1181-1184, 2001.

[21] R. H. Selzer, W. J. Mack, P. L. Lee, H. Kwong-Fu, and H. N. Hodis, "Improved common carotid elasticity and intima-media thickness measurements from computer analysis of sequential ultrasound frames," Atherosclerosis, vol. 154, no. 1, pp. 185-193, 2001.

[22] H. Kanai, H. Hasegawa, M. Ichiki, F. Tezuka, and Y. Koiwa, "Elasticity imaging of atheroma with transcutaneous ultrasound: preliminary study," Circulation, vol. 107, no. 24, pp. 3018-3021, 2003.

[23] C. Stephanis, D. Mourmouras, and D. Tsagadopoulos, "On the elastic properties of arteries," Journal of Biomechanics, vol. 36, no. 11, pp. 17271731, 2003. 
[24] P. Boutouyrie, D. P. Germain, J.-N. Fiessinger, B. Laloux, J. Perdu, and S. Laurent, "Increased Carotid Wall Stress in Vascular Ehlers-Danlos Syndrome," Circulation, vol. 109, no. 12, pp. 1530-1535, 2004.

[25] H. Hasegawa and H. Kanai, "Measurement of Elastic Moduli of the Arterial Wall at Multiple Frequencies by Remote Actuation for Assessment of Viscoelasticity," Japanese Journal of Applied Physics, vol. 43, no. 5B, pp. 3197-3203, 2004.

[26] I. Masson, "Contribution à la modélisation mécanique du comportement dynamique hyperélastrique et anisotrope de la paroi artérielle," Thèse de doctorat, Université Paris 12 Val de Marne, 2008.

[27] S. Avril, M. Bonnet, A.-S. Bretelle, M. Grédiac, F. Hild, P. Ienny, F. Latourte, D. Lemosse, S. Pagano, E. Pagnacco, and F. Pierron, "Overview of Identification Methods of Mechanical Parameters Based on Full-field Measurements," Experimental Mechanics, vol. 48, no. 4, pp. 381-402, 2008.

[28] K. B. Chandran, J. H. Mun, K. K. Choi, J. S. Chen, A. Hamilton, A. Nagaraj, and D. D. McPherson, "A method for in-vivo analysis for regional arterial wall material property alterations with atherosclerosis: preliminary results," Medical Engineering \& Physics, vol. 25, no. 4, pp. 289-298, 2003.

[29] A. J. Hamilton, H. Kim, A. Nagaraj, J.-H. H. Mun, L. L. Yan, S. I. Roth, D. D. McPherson, and K. B. Chandran, "Regional material property alterations in porcine femoral arteries with atheroma development," Journal of Biomechanics, vol. 38, no. 12, pp. 2354-2364, 2005.

[30] R. A. Baldewsing, M. G. Danilouchkine, F. Mastik, J. A. Shaar, P. W. Serruys, and A. F. W. van der Steen, "An Inverse Method for Imaging the Local Elasticity of Atherosclerotic Coronary Plaques," IEEE Transactions on Information Technology in Biomedicine, vol. 12, no. 3, pp. 277-289, 2008.

[31] S. Le Floc'h, J. Ohayon, P. Tracqui, G. Finet, A. M. Gharib, R. L. Maurice, G. Cloutier, and R. I. Pettigrew, "Vulnerable Atherosclerotic Plaque Elasticity Reconstruction Based on a Segmentation-driven Optimization Procedure using Strain Measurements: Theoretical Framework," IEEE Transactions on Medical Imaging, vol. 28, no. 7, pp. 1126-1137, 2009. 
[32] A. P. Lin, E. Bennett, L. E. Wisk, M. Gharib, S. E. Fraser, H. Wen, and E. Benett, "Circumferential Strain in the Wall of the Common Carotid Artery: Comparing Displacement-Encoded and Cine MRI in Volunteers," Magnetic resonance in Medicine, vol. 60, no. 1, pp. 8-13, 2008.

[33] M. I. Miga, "A new approach to elastography using mutual information and finite elements," Physics in Medicine and Biology, vol. 48, no. 4, pp. 467-480, 2003.

[34] C. W. Washington and M. I. Miga, "Modality independent elastography (MIE): a new approach to elasticity imaging." IEEE Transactions on Medical Imaging, vol. 23, no. 9, pp. 1117-1128, 2004.

[35] M. A. Bernstein, K. F. King, and X. J. Zhou, Handbook of MRI pulse sequences. Elsevier Inc., 2004.

[36] F. Guyon and R. Le Riche, "Least Squares Parameter Estimation and the Levenberg-Marquardt Algorithm : Deterministic Analysis, Sensitivities and Numerical Experiments," Institut National des Sciences Appliquées, Tech. Rep., 2000.

[37] A. Franquet, S. Avril, R. Le Riche, and P. Badel, "Identification of heterogeneous elastic properties in stenosed arteries: a numerical plane strain study," Computer Methods in Biomechanics and Biomedical Engineering, vol. 15, no. 1, pp. 49-58, 2012.

[38] ABAQUS 6.8, Abaqus Theory Manual, Dassault Systèmes Simulia Corp. Providence (RI,USA), Ed., 2008.

[39] M. R. Skilton, L. Boussel, F. Bonnet, S. Bernard, P. C. Douek, P. Moulin, and A. Serusclat, "Carotid intimamedia and adventitial thickening: Comparison of new and established ultrasound and magnetic resonance imaging techniques," Atherosclerosis, vol. 21, pp. 405-410, 2011.

[40] I. S. Mackenzie, I. B. Wilkinson, and J. R. Cockcroft, "Assessment of Arterial Stiffness in Clinical Practice," QJM, vol. 95, no. 2, pp. 67-74, 2002.

[41] S. Laurent, J. Cockcroft, L. Van Bortel, P. Boutouyrie, C. Giannattasio, D. Hayoz, B. Pannier, C. Vlachopoulos, I. Wilkinson, and H. StruijkerBoudier, "Expert Consensus Document on Arterial Stiffness: Method- 
ological Issues and Clinical Applications," European Heart Journal, vol. 27, no. 21, pp. 2588-2605, 2006.

[42] R. A. Baldewsing, J. A. Schaar, F. Mastik, C. W. J. Oomens, and A. F. W. van der Steen, "Assessment of vulnerable plaque composition by matching the deformation of a parametric plaque model to measured plaque deformation," IEEE Transactions on Medical Imaging, vol. 24, no. 4, pp. 514528, 2005.

[43] V. Taviani, M. P. F. Sutcliffe, P. Wong, Z.-Y. Li, V. Young, M. J. Graves, and J. H. Gillard, "In vivo non-invasive high resolution MR-based method for the determination of the elastic modulus of arterial vessels," Annual International Conference of the IEEE Engineering in Medicine and Biology Society., vol. 2008, pp. 5569-5572, 2008.

[44] G. A. Holzapfel and H. W. Weizsacker, "Biomechanical behavior of the arterial wall and its numerical characterization," Computers in Biology and Medicine, vol. 28, no. 4, pp. 377-392, 1998.

[45] J. D. Humphrey, Cardiovascular Solid Mechanics : Cells, Tissues and Organs. New-York: Springer-Verlag, 2002.

[46] A. I. Veress, N. Phatak, and J. A. Weiss, "Deformable Image Registration with Hyperelastic Warping," in Handbook of Biomedical Image Analysis, 3rd ed., J. S. Suri, D. L. Wilson, and S. Laxminarayan, Eds. New-York: Kluwer Academic/Plenum, 2004, pp. 487-534.

[47] J. A. Weiss and A. I. Veress, "Strain measurement using deformable image registration," in Mechanics of Biological Tissue, G. A. Holzapfel and R. W. Ogden, Eds., 2006, pp. 489-501.

[48] N. S. Phatak, S. A. Maas, A. I. Veress, N. A. Pack, E. V. R. Di Bella, and J. A. Weiss, "Strain measurmeent in the left ventricule during systoo le with deformable image registration," Medical Image Analysis, vol. 13, no. 2, pp. 354-361, 2009. 